Results The search identified 6,611 unique citations. Further references may be identified by hand-searching references of included articles and contacting authors. Title and abstract screening has identified 278 potentially relevant papers so far.

Conclusions Full text review of relevant citations is underway. The review will be completed by autumn 2019. The results will document how ED has been evaluated in this setting and pinpoint what elements may prove most effective and in what context. This will inform future research in the development and assessment of ED-based psychotherapeutic interventions in the palliative care setting.

\section{THE PLACE OR THE ENVIRONMENT: WHAT IS A MORE PREFERRED OPTION AT THE END OF LIFE FOR PEOPLE IN WALES?}

Ishrat Islam*, Anthony Byrne, Annmarie Nelson. Cardiff University, UK

10.1136/spcare-2019-mariecuriepalliativecare.4

Introduction Public preference to die at home has been well documented in the literature (Schou-Andersen et al., 2016). An indicator of measuring success of End of Life Care Delivery Plan of Welsh Government is the proportion of patients who are cared for in their preferred places(Welsh Government, 2017). Uncertainties around accessing facilities to meet clinical and psycho-social need may have influence on changing public preference.

Aim To identify public preferences at end of life.

Method Online public survey using JISC tool. Data were analysed using descriptive statistics and thematic approach for this mixed method study.

Results 2210 people (Age: Mean+SD; 55+14 yrs) participated in the survey $(29 \%$ were patients/cares, $23 \%$ academic/health professionals and $48 \%$ with no caregiving/bereavement experience) Only $31 \%$ of total surveyed believed that EoLC facilities were accessible and $49 \%$ thought these facilities were insufficient to meet the need. More people preferred to be surrounded by loved ones (62\%); having access to trained professionals (84\%), die in dignity (55\%); be peaceful $(40 \%)$; be symptom-free $(30 \%)$, take part in care decision $(26 \%)$ than those who preferred to be at home (24\%). Preferred services and environment were expressed with free text by 189 and 87 respondents. Most people preferred their choices being respected; a large portion advocated for euthanasia. Pain management and having access to trained professionals came as preferred options by many but very few people preferred being at home.

Conclusions Access to trained support and having loved ones around them in a peaceful dignified environment outweigh the importance of a physical place of care.

\section{UNDERSTANDING THE NEEDS OF DIVERSE COMMUNITIES IN PALLIATIVE CARE}

Monica Reardon*. Marie Curie, Wales and Southern England, UK

10.1136/spcare-2019-mariecuriepalliativecare.5

Current estimates suggest 1 in 4 people miss out on palliative care. ${ }^{1}$ Older people, people with non-cancer conditions, from BAME backgrounds, or with learning disabilities are some groups that face barriers to good end of life care. ${ }^{2}$ With more people living and dying from complex chronic illnesses ${ }^{3}$ and estimates that by $205120-30 \%$ of the UK population will be ethnic minorities ${ }^{4}$, these groups' needs will significantly influence the growth in future palliative care demand.

2 projects in South Wales explored these barriers for people:

- from BAME backgrounds;

- with dementia;

- with learning disabilities; and

- from religious and non-religious backgrounds

We consulted 499 people through focus groups and interviews.

Findings

- People's understanding of palliative care is limited. Accessible and tailored communication would reduce fear and increase trust in services.

- People with non-cancer conditions and from BAME backgrounds feel they are less likely to be referred to palliative care. Some reported experiencing discrimination. Less restrictive referral processes would reduce barriers.

- People decline services for fear that their spiritual, cultural, or diagnosis-related needs will not be met. Staff need increased understanding of diverse needs and skills to meet them, and this inclusion should be promoted within communities.

As palliative care need increases and demographics shift, services will support an increasingly diverse patient population. It is vital that we work in partnership across the health and social care system to reduce systemic barriers to access services. We must also recognise the value of communities, and the experience and knowledge they have, to help us improve.

\section{REFERENCES}

1. Hospice UK. (2018) Hospice care in Wales 2018: supporting people closer to home. Available at: https://www.hospiceuk.org/what-we-offer/publications (Accessed 02 July 2019).

2. Care Quality Commission. (2016) A different ending: Addressing inequalities in end of life care. Available at: https://www.cqc.org.uk/publications/themed-work/ different-ending-end-life-care-review (Accessed 02 July 2019).

3. Marie Curie. (2016) Palliative care and the UK nations, implications for Wales. Available at: https://www.mariecurie.org.uk/policy/publications (Accessed 02 July 2019).

4. Sunak R, Rajeswaran S. (2014) A Portrait of Modern Britain. Policy Exchange. Available at: https://policyexchange.org.uk/wp-content/uploads/2016/09/a-portraitof-modern-britain.pdf (Acessed 02 July 2019).

\section{BREAKING BAD NEWS TRAINING: LESSONS FROM OUTSIDE HEALTHCARE}

${ }^{1}$ Gehan Soosaipillai* ${ }^{2}$ Stephanie Archer, ${ }^{1}$ Anna-Maria Bielinska, ${ }^{1}$ Hutan Ashrafian, ${ }^{1}$ Ara Darzi. ${ }^{1}$ Imperial College London, UK; ${ }^{2}$ University of Cambridge, UK

10.1136/spcare-2019-mariecuriepalliativecare.6

Healthcare workers frequently break bad news, but often lack formal training. Other job sectors (e.g. military and human resources) also break bad news on a regular basis. Lessons from these sectors may be valuable when developing training resources within healthcare. 
Aim To understand how those who work outside of healthcare are trained to break bad news.

Method Using a snowball approach, a convenience sample of 20 participants from a range of sectors were invited to participate. Twelve completed semi-structured interviews (see table 1). Audio recordings were transcribed, anonymised and subjected to thematic analysis.

Results Though formal training was lacking in many sectors, the military, in particular, provided rigorous and consistent training courses for their Notification and Visiting Officers. Experiential learning by observing others or through own practice, or interactive learning in a safe and simulated environment (e.g. role-play) was common. Some sectors recognised the challenge of breaking bad news and have installed supportive measures. Digital learning was seen as 'the way forward', with accessibility and flexibility that matches modern working styles. There was a demand for more learning on breaking bad news, even in a digital medium, but there was little recognition of such resources being available.

\begin{tabular}{lc} 
Abstract 6 Table 1 & Participant demographics \\
\hline Variable & Participants (n=12) \\
\hline Gender & 4 \\
Male & 8 \\
Female & Male to female ratio (1:2) \\
& \\
Age & 0 \\
$18-24$ & 2 \\
$25-34$ & 6 \\
$35-44$ & 2 \\
$45-54$ & 2 \\
$55-64$ & \\
Sector & \\
Information technology & 1 \\
Law enforcement and security & 4 \\
Public services and administration & 2 \\
Recruitment and HR & 5 \\
\hline
\end{tabular}

Conclusion Other job sectors apply similar learning modalities to healthcare but place greater emphasis on training their workforce in breaking bad news. There is a need for research studying enhanced training for breaking bad news in healthcare by focusing on preparedness and support as this would likely offer comparable benefits for the healthcare sector.

\section{Posters}

\section{A QUALITATIVE STUDY OF GENERAL PRACTITIONERS' DECISIONS ABOUT PRESCRIBING ANTICIPATORY MEDICINES AT THE END OF LIFE}

${ }^{1}$ Ben Bowers, ${ }^{2}$ Kristian Pollock, ${ }^{1}$ Sam Barclay, ${ }^{2}$ Stephen Barclay. ${ }^{1}$ University of Cambridge, UK; ${ }^{2}$ University of Nottingham, UK

\subsection{6/spcare-2019-mariecuriepalliativecare.7}

Background General Practitioners (GPs) have a central role in decisions about prescribing anticipatory medications (AMs) to help control symptoms at the end of life. (Faull, et al. 2013)
Little is known about GPs' decision-making processes in prescribing AMs and the subsequent use of prescribed drugs.

Aim To explore GPs decision-making processes in the prescribing and use of AMs for patients at the end of life.

Method A qualitative interpretive descriptive enquiry with a purposive sample of thirteen GPs working across one English county. Data was collected in 2017 via semi-structured interviews and analysed inductively using Braun and Clarke's thematic analysis.

Results Three themes were constructed from the data: 1) 'Something we can do'. AMs were a tangible intervention GPs felt they could offer to provide symptom relief for patients approaching death. 2) 'Getting the timing right'. The prescribing of AMs was recognised as a harbinger of death for patients and families. GPs preferred to prescribe drugs weeks before death was expected, while recognising this meant that many prescribed AMs were never used. 3) 'Delegating care whilst retaining accountability'. GPs relied on nurse to assess when to administer drugs and keep them updated about their use.

Conclusion GPs view AMs as key to symptom management for dying people. AMs are routinely prescribed even though they are often not used. In order to feel comfortable delegating care, GPs need regular access to nurses and trust in their skills to administer drugs appropriately. Patient and family experiences of AMs warrant urgent investigation.

\section{BATTERY-OPERATED FAN AND CHRONIC BREATHLESSNESS: DOES IT HELP?}

${ }^{1}$ Matilda Barnes-Harris, ${ }^{1}$ Miriam Johnson, ${ }^{2}$ Flavia Swan, ${ }^{3}$ Victoria Allgar, 'Sara Booth, ${ }^{4}$ David Currow, ${ }^{2}$ Simon Hart, ${ }^{4}$ Jane Phillips. 'Wolfson Palliative Care Research Centre, Hull York Medical School, UK; ${ }^{2}$ Hull York Medical School, University of York, UK; ${ }^{3}$ Department. of Oncology, University of Cambridge, UK: ${ }^{4}$ IMPACCT, Faculty of Health, University of Technology Sydney, Australia

\subsection{6/spcare-2019-mariecuriepalliativecare.8}

Objectives To examine whether use of a hand-held fan ('fan') improves breathlessness and increases physical activity.

Methods A secondary exploratory analysis using pooled data from the fan arms of two feasibility randomised controlled trials in people with chronic breathlessness; i) fan and activity advice $v s$ activity advice, ii) activity advice alone or with the addition or the 'calming hand', or the fan, or both. Descriptive statistics and regression analysis to explore patient characteristics associated with benefit (e.g. age, sex, diagnosis, general self-efficacy).

Results Forty-one participants were allocated the fan (73 years [IQR 65-76, range 46-88], 59\% male, 20 (49\%) chronic obstructive pulmonary disease (COPD), three $(7 \%)$ heart failure, three (7\%) cancer). Thirty-five $(85 \%)$ reported that the fan helped breathing, and 22 (54\%) reported increased physical activity. Breathlessness benefit was more likely in older people, those with COPD and those with a carer. However, due to the small sample size none of these findings were statistically significant. Those with COPD were more likely to use the fan than people with other diagnoses (Odds Ratio 5.94 [95\% CI (95\%CI) 0.63, 56.21, $\mathrm{p}=0.120)$ ] (table 1).

Conclusions These exploratory data support that the fan helps chronic breathlessness in most people and adds new data to indicate that the fan is perceived to increase people's 